\title{
Do we live in the best of all possible worlds? The fine-tuning of the constants of nature
}

\author{
Thomas Naumann ${ }^{1, a}$ \\ ${ }^{1}$ Deutsches Elektronen-Synchrotron DESY, Platanenallee 6, 15738 Zeuthen, Germany
}

\begin{abstract}
Our existence depends on a variety of constants which appear to be extremely fine-tuned to allow for the existence of life. These include the number of spatial dimensions, the strengths of the forces, the masses of the particles, the composition of the Universe and others. On the occasion of the $300^{\text {th }}$ anniversary of the death of G.W. Leibniz we discuss the question of whether we live in the "Best of all Worlds". The hypothesis of a multiverse could explain the mysterious fine tuning of so many fundamental quantities. Anthropic arguments are critically reviewed.
\end{abstract}

\section{The best of all worlds - From Leibniz to Einstein}

The Electress of Brandenburg and later Queen of Prussia Sophie Charlotte was a lifelong friend to Gottfried Wilhelm Leibniz who died 300 years ago. They inspired the king to create the Kurfürstlich Brandenburgische Societät der Wissenschaften zu Berlin in 1700. Leibniz became the first lifetime President of this academy renamed in 1701 to Royal Prussian Society of Sciences and known today as Berlin-Brandenburgische Akademie der Wissenschaften.

Since Leibniz tended to never finish his many activities and to spread his ideas over thousands of loose notes the Queen asked him to summarize his world view in a popular pamphlet. As he was very much indebted to her he completed in 1710 the only coherent text published during his lifetime, his "Essays on the Goodness of God, the Freedom of Man and the Origin of Evil" $[1,2]$.

In this treatise he tried what one might call the impossible: to unify and harmonise science, metaphysics and theology. In $\S 8$ of its first part he writes: „this supreme wisdom, united to a goodness that is no less infinite, cannot but have chosen the best. ... if there were not the best among all possible worlds, God would not have produced any... there is an infinitude of possible worlds among which God must have chosen the best, since he does nothing without acting in accordance with supreme reason..." [2]. The important point for modern science is that Leibniz assumes the existence of a multitude of possible worlds although by this he meant imaginary or intended but not real worlds.

In modern physics it was Einstein who in a discussion with his assistant Banesh Hoffmann in the late thirties asked a similar question: "When I am judging a theory, I ask myself whether, if I were God, I would have arranged the world in such a way." [3] And to Ernst Gabor Straus, his assistant from 1940-48, Einstein made the famous remark: "What

\footnotetext{
${ }^{\text {a }}$ Corresponding author: Thomas.Naumann@desy.de
} 
really interests me is whether God could have created the world any differently." [4] This reiterates Leibniz's question whether we live in the best of all worlds in the context of modern physics.

\section{Had God a choice?}

An early observation of the fine-tuning of the fundamental cosmological parameters (Hubble and gravitational constants $\mathrm{H}_{0}$ and $\mathrm{G}_{\mathrm{N}}$ and mean density $\Omega$ of the Universe) was made by Dicke in 1961 [5]. If any of several fundamental physical constants were only slightly different, the Universe would be unlikely to lead to the formation of nuclear, atomic or cosmic structures which allow for chemical elements, stellar burning and finally life.

The Universe indeed appears extremely fine-tuned for life. We will illustrate this phenomenon by asking the question: "What if we change some of the constants of nature?" proceeding from the most general to more specific constants:

- the dimensionality of the Universe

- symmetries: the matter-antimatter asymmetry

- the cosmic inventory: mean density, Hubble and cosmological constant, curvature

- What if no Higgs? The masses of weak bosons, quarks and the electron.

The amazing fine-tuning of nuclear processes visible in the deuterium bottleneck, the triple-alpha process of stellar burning and nucleon-nucleon binding has to be left for a separate discussion.

\subsection{The dimensionality of the Universe}

A world with less than three dimensions was discussed by Barrow and Tipler [6] and Hawking [7]. In such a world, the digestive tract of any higher animal would separate it into disjoint upper and lower parts. Also nerves and blood vessels would cross in two dimensions, and food absorption would have to proceed by breaking a one-dimensional boundary.

The case of a world with more than three spatial dimensions was discussed by Ehrenfest in $1917[8,9]$. He considered the scattering of a small on a large mass. The probe particle is either trapped by the large mass - or it escapes to infinity. In more than three dimensions no stable orbits of planets are possible! The same is true for electrons in classical atoms. In 1963 Tangherlini [10] showed that also in quantum mechanics in more than three spatial dimensions there are no stable atoms.

\subsection{The matter-antimatter asymmetry}

We have to assume that the Universe is neutral and that all charges are conserved. Hence the Big Bang should have started from equal amounts of matter and antimatter. A matterantimatter symmetric universe would however have rapidly annihilated to an empty light bubble while we live today in a matter dominated Universe. Related to the density of relic photons the number of matter particles amounts only to about one in several billions. The matter content of our Universe is hence characterized by a tiny but decisive misbalance. A significantly larger matter density would have caused a rapid recollapse of the Universe without any chance for structure formation and life.

\subsection{The cosmic inventory}


As already observed by Dicke in 1961 [5] the evolution of the Universe is characterized by a delicate balance of its inventory, a balance between repulsion and attraction, between expansion and contraction. Since gravity is purely attractive its action sums up throughout the whole Universe. The strength of this attraction is defined by the gravitational constant $\mathrm{G}_{\mathrm{N}}$ and by an environmental parameter, the density $\Omega_{\mathrm{M}}$ of (dark and baryonic) matter. The strength of the repulsion is defined by two parameters: the initial impetus of the Big Bang parameterized by the Hubble constant $\mathrm{H}_{0}$ and the cosmological constant $\Lambda$.

As determined from data of the PLANCK satellite combined with other cosmological observations the total density of the Universe is $\Omega_{\text {tot }}=\rho / \rho_{\text {crit }}=1.0002 \pm 0.0026$ and thus extremely close to its critical density $\rho_{\text {crit }}=3 \mathrm{H}_{0}{ }^{2} / 8 \pi \mathrm{G}_{\mathrm{N}}$ with a contribution from a curvature of the Universe of $\Omega_{\mathrm{k}}=0.000 \pm 0.005$ [11]. However, the balance between the ingredients of the Universe is time-dependent. Any deviation from zero curvature after inflation is amplified by many orders of magnitude. Hence, a cosmic density fine-tuned to flatness today to less than a per mille must have been initially fine-tuned to tens of orders of magnitude.

Another mystery is the smallness of the cosmological constant. First of all, the Planck scale is the only natural energy scale of gravitation: $\mathrm{m}_{\mathrm{Pl}}=\left(\hbar \mathrm{c} / \mathrm{G}_{\mathrm{N}}\right)^{1 / 2}=1.2 \cdot 10^{19} \mathrm{GeV} / \mathrm{c}^{2}$. Compared to this natural scale the cosmological constant or dark energy density $\Lambda$ is tiny:

$$
\Lambda \sim(10 \mathrm{meV})^{4} \sim\left(10^{-30} \mathrm{~m}_{P l}\right)^{4}=10^{-120} \mathrm{~m}_{P l}{ }^{4} .
$$

The cosmological constant is also much smaller than expected from the vacuum expectation value of the Higgs field which like the inflaton field or dark energy is an omnipresent scalar field:

$$
\left\langle\Phi^{4}\right\rangle \sim m_{H}^{4} \sim(100 \mathrm{GeV})^{4} \sim 10^{52} \Lambda \quad .
$$

As observed by Weinberg in 1987 there is an 'anthropic' upper bound on the cosmological constant $\Lambda$. He argued "that in universes that do not recollapse, the only such bound on $\Lambda$ is that it should not be so large as to prevent the formation of gravitationally bound states." [12]

\subsection{What if no Higgs? The fine-tuning of particle masses}

\subsubsection{Boson masses}

The carriers of the strong and electromagnetic interaction, the gluon and the photon, are massless. After spontaneous breaking of the electroweak symmetry the weak bosons acquire a mass which at low energies suppresses the weak interaction. Without this symmetry breaking, i.e. without a Higgs field, the weak bosons would be massless, and the weak interaction would be about as strong as the electromagnetic one. Star burning would not last billions of years but rather resemble a nuclear explosion with no time left for the evolution of planetary systems and life.

On a more quantitative level the mass $\mathrm{m}_{\mathrm{W}}=80.4 \mathrm{GeV}$ of the $\mathrm{W}$ bosons governs the fusion process $\mathrm{pp} \rightarrow \mathrm{d} \mathrm{e}^{+} \mathrm{v}_{\mathrm{e}}$ in stars. The cross section of this fusion process which corresponds to inverse beta decay depends on the inverse of the fourth power of the weak boson mass: $\sigma \sim\left(\mathrm{m}_{\mathrm{W}}\right)^{-4}$. An increase of the weak boson mass by a factor of two would let the Sun and similar stars burn out within roughly a billion years. Such a rapid star burning would make the evolution of higher life almost impossible.

\subsubsection{Fermion masses}


The neutron is heavier than the proton and hence unstable: $\mathrm{m}_{\mathrm{n}}-\mathrm{m}_{\mathrm{p}}=1.3 \mathrm{MeV} \approx 1 \% \mathrm{o}$ $\mathrm{m}_{\text {nucleon}}$. This is due to the difference of 3-4 MeV between the masses of the down and up quark (this difference is partially compensated by the stronger electric repulsion of the additional up quark in the proton). In the second and third quark families, however, the up quarks are significantly heavier than the down quarks. So one might ask: What if also in the first family the up quark would be heavier than the down quark?

Then the proton would be heavier than the neutron and decay: $\mathrm{p} \rightarrow \mathrm{n} \mathrm{e} \mathrm{e}^{+} v_{\mathrm{e}}$. The positron would subsequently annihilate with an electron $\mathrm{e}^{+} \mathrm{e}^{-} \rightarrow \gamma \gamma$. The result would be a neutral universe consisting of photons, neutrons and neutrinos without a chance for the formation of nuclei and atoms and hence life. Since $m_{p}-\left(m_{n}+m_{e}\right)>2.2 \mathrm{MeV}=E_{b}{ }^{d}$ the free energy of this decay is larger than the binding energy $\mathrm{E}_{\mathrm{b}}{ }^{\mathrm{d}}$ of the neutron. This also makes the deuteron unstable $\mathrm{d} \rightarrow 2 \mathrm{n} \mathrm{e}^{+} v_{\mathrm{e}}$ so that the decaying protons cannot be trapped in deuterium and form helium like in our world.

Since the Rydberg energy is $R=0.5 \boldsymbol{\alpha}^{2} \mathrm{~m}_{\mathrm{e}}$ and the Bohr radius $r_{B}=1 /\left(\boldsymbol{\alpha} \mathrm{m}_{\mathrm{e}}\right)$ the mass of the electron governs energy levels and distances of the atomic world. An increase of the electron mass to $5 \mathrm{MeV}$ would have the same effect as making the up quark heavier than the down quark. Protons would decay, and we would end up in a neutral world with only photons, neutrons and neutrinos left over.

\section{The anthropic principle}

The observation of the fine-tuning of the Universe is closely related to the anthropic principle. An early expression of this principle was done by Dicke in 1961: "The existence of physicists... [is] sufficient to demand that... relations between the three numbers $\left[\mathrm{G}_{\mathrm{N}}\right.$, $\left.\mathrm{H}_{0}, \Omega\right]$ be satisfied." Lord Martin Rees, cosmologist and Astronomer Royal in his popular book "Just Six Numbers" [13] added the number of dimensions and the relative strength of the electromagnetic to the gravitational interaction to his discussion of the fine tuning of the constants of nature. The subtitle of the German translation nicely summarizes this book: "Hatte Gott eine Wahl?" - "Had God a choice?"

In 1986 in their ground-breaking book "The Anthropic Cosmological Principle" [6] Barrow and Tipler explain: this principle holds that the fundamental structure of the Universe is determined by the existence of intelligent observers. The Universe is as it is because if it were otherwise, observers could not exist. In short: Man not only fits to the Universe. The Universe also fits to man. Hawking in his "Brief History of Time" pointed out in 1988: "The remarkable fact is that the values of these numbers seem to have been very finely adjusted to make possible the development of life." [14]

The anthropic principle has been under intense discussions and criticism over the last decades. Steven Weinberg who repeatedly referred to it reportedly warned: "A physicist talking about the anthropic principle runs the same risk as a cleric talking about pornography: No matter how much you say you are against it, some people will think you are a little too interested." [15]

The big epistemological problem behind the anthropic principle is that for the moment we only observe one Universe. Hence, we cannot apply the scientific method to make repeated experiments with the object of our investigation in order to extract regularities and laws. The anthropic principle also runs the danger to end up in a tautology or a causal circle: We only observe universes that allow an observer. Another critical point is that the anthropic principle makes no predictions but only postdictions.

Another open question should be carefully considered. What exactly is fine-tuned in our Universe: underlying laws or parameters, and are the parameters fundamental or environmental ones? 


\section{From Universe to Multiverse}

The problem of the uniqueness of our Universe can be avoided going from a single and unique universe to a multiverse consisting of a huge number of different universes. At the extreme energy densities at Planck time $\left(\sim 10^{-43} \mathrm{~s}\right)$ immediately after the Big Bang due to quantum gravity the Universe is thought to be dominated by vacuum fluctuations of spacetime. Soon $\left(\sim 10^{-36} \mathrm{~s}\right)$ after, in the cosmological model of inflation vacuum fluctuations of a scalar (Higgs-like) inflaton field of the size of a tiny fraction of a proton radius could have inflated by many orders of magnitude to a region larger than the event horizon which later expanded to today's observable Universe.

At these extreme energies all non-gravitational interactions are still thought to be unified to one primordial force. At decreasing energies these grand unified symmetries can break down to the symmetries of separate forces in many different ways. In our world we observe $\mathrm{SU}(3)_{\mathrm{C}}, \mathrm{SU}(2)_{\mathrm{W}}$ and $\mathrm{U}(1)$. On top of that the superstring theory developed in the 1980ies by Green, Schwarz, Witten and others [16] assumes six (or seven) extra spatial dimensions which are curled up at the small distance scales relevant in the early Universe. These theories give rise to a huge $\left(\sim 10^{500}\right)$ [17] number of possible compactifications to the three dimensions which we observe at the low energies in our Universe today.

Each of the inflationary bubbles may have evolved into a universe with a different realization of the physics potential hidden in grand unified and superstring theories. So the physics in these universes can be different from the physics in our Universe. This vast field of possibilities Susskind called "The anthropic landscape of string theory" [18].

In his essay "Particle Physics and Inflationary Cosmology" [19] Linde who in the 1980ies together with Guth [20], Steinhardt and others had developed the inflationary scenario explained: "Instead of a Universe with a single law of physics operating everywhere we are discussing an eternally existing self-reproducing Multiverse which consists of many different parts where all possibilities can be realized." Applying the anthropic principle to the string landscape Weinberg in 2007 in an article "Living in the Multiverse" [21] stated that this principle "may explain how the constants of nature that we observe can take values suitable for life without being fine-tuned by a benevolent creator."

Steinhardt however criticized that no experiment can rule out the multiverse hypothesis since it allows for all possible physics scenarios: “...the inflationary paradigm is so flexible that it is immune to experimental and observational tests. First, inflation is driven by a hypothetical scalar field, the inflaton, which has properties that can be adjusted to produce effectively any outcome. Second, inflation does not end with a universe with uniform properties, but almost inevitably leads to a multiverse with an infinite number of bubbles, in which the cosmic and physical properties vary from bubble to bubble. The part of the multiverse that we observe corresponds to a piece of just one such bubble. Scanning over all possible bubbles in the multiverse, everything that can physically happen does happen an infinite number of times. No experiment can rule out a theory that allows for all possible outcomes. Hence, the paradigm of inflation is unfalsifiable." [22]

In his discussion „Universe Or universe? It All Depends On The Multiverse“ of whether to capitalize the word 'Universe' Gleiser [23] mentions that "we may obtain information of neighbouring universes, if they have collided with ours in the past" [24] but also insists that we have only our Universe.

\subsection{Learning from history: Kepler's Harmonices Mundi}

In a similar spirit as Kepler at his time asked the question: "Why are there just six planets?" we ask today: "Why are there just three fermion families?" Without deeper knowledge it is impossible to decide whether such a question is a fundamental or an environmental one. In 
his main works Mysterium Cosmographicum (1596) and Harmonices Mundi (1619) Kepler was looking for God's plan of the geometry of the Universe and tried to explain the ratios of the planetary orbits from first (and divine) principles. Nesting and encasing the spheres inscribed and circumscribed to the five Platonic solids within one another he produced six shells the radii of which miraculously corresponded to the sizes of the orbits of the six known planets.

Since Kepler did not know that there are more planets in our solar system, millions of planetary systems in our galaxy and billions of galaxies in our Universe he considered an accidental fine-tuning to be fundamental. He thus tried to answer an irrelevant question. Also, Kepler's model relied on the orbits being circles which clearly contradicted his most important discovery that the orbits are ellipses. To save God's plan Kepler had to compromise his Platonic model.

So a sufficient number of alternatives can easily solve an apparent fine-tuning problem. The same may be true for our present anthropic problem: We are fascinated by our finetuned Universe which may be completely accidental in view of a sufficiently large number of alternative universes.

Kepler's dilemma, however, besides the statistical solution also had a fundamental solution: based on his laws Newton was able to create his fundamental theory of gravity. So in Kepler's case two solutions of the dilemma existed which were both viable and even not contradicted each other: the statistical and the fundamental one.

We may be in a similar situation today. The existence within a multiverse of a large number of universes with different dimensionalities, symmetry breaking schemes, strengths of the fundamental forces, particle masses, inflation scenarios and asymmetries (like matter-antimatter) may allow for exactly the fundamental parameters needed for the formation of stable structures, chemical elements and finally life in our world. Fine-tuning would then be a result of a natural cosmic selection of our world out of many worlds as the best habitat for the development of life (see e.g. Smolin [25]). Or as Gell-Mann once put it: "If we really live in a multiverse, physics will have been reduced to an environmental science like botany." [26]

So the indefiniteness and openness which Steinhardt and others deplore become very productive: they open the rich field of possibilities needed for the development of life.

\subsection{From physics to metaphysics: the ultimate dethronement of Man}

The physics in our world could thus be one of many solutions of more general physical laws. Based on his treatise Physics Aristotle subsequently composed his Metaphysics. In a similar spirit we might consider the physics of a multiverse as a meta-physics in its original positive sense: a meta-physics encompassing many possible physics scenarios realized in a huge number of universes of a multiverse.

Copernicus assumed that the Earth is not at the center of the Universe but only one of many planets orbiting around the Sun. Later Darwin proposed that Man is not the crown and goal of creation but an animal like others and a product of biological evolution. Hubble showed that our Milky Way is only one out of many galaxies in the Universe. Modern science repeatedly pushed Man off his throne into some remote corner of the Universe. Today, we may get rid of the anthropic problem assuming that our Universe is only one out of a multitude of universes in a multiverse.

This would be the ultimate dethronement of Man in the history of science. Monod described this aspect of human existence as follows: "The ancient alliance has been broken. Man at last knows he is alone in the unfeeling immensity of the Universe, out of which he has emerged only by chance. His destiny is nowhere spelled out, nor is his duty. The kingdom above or the darkness below; it is for him to choose." [27] 
Finally, a word of caution is needed. The multiverse is not an established theory but a hypothesis. Asking the question "Does the Multiverse Really Exist?" the astrophysicist George Ellis said [28]: "As skeptical as I am, I think the contemplation of the multiverse is an excellent opportunity to reflect on the nature of science and on the ultimate nature of existence: why we are here... In looking at this concept, we need an open mind, though not too open. It is a delicate path to tread. Parallel universes may or may not exist; the case is unproved. We are going to have to live with that uncertainty. Nothing is wrong with scientifically based philosophical speculation, which is what multiverse proposals are. But we should name it for what it is."

Hypotheses have been very fruitful in the history of science, and it may take a lot of time and effort to verify or falsify them. The history of the atomic hypothesis is a good example. Based on his observation of the law of multiple proportions in 1808 John Dalton in his essay „A New System of Chemical Philosophy“ formulated the hypothesis that all matter consists of indivisible atoms. The final confirmation of the atomic hypothesis took almost one century, and the theories of Maxwell, Boltzmann and Einstein had to stand against the criticism of positivists like Mach.

In order to keep science alive we should therefore aim to keep an open mind for hypotheses even if we cannot today give prescriptions how to verify or falsify them.

\section{References}

1. G. W. Leibniz, Essais de théodicée sur la bonté de Dieu, la liberté de l'homme, et l'origine du mal (Mortier, Amsterdam, 1710)

2. G. W. Leibniz, Essays on the Goodness of God, the Freedom of Man and the Origin of Evil. In: The Philosophical Works of G.W. Leibniz, Translated by E.M. Huggard from C.J. Gerhardt, Die Philosophischen Schriften von G.W. Leibniz, Berlin 1875-90; (Tuttle, Morehouse \& Taylor, New Haven, 1890)

3. B. Hoffmann, quoted in: A. Calaprice, ed., The Ultimate Quotable Einstein, p. 407 (Princeton University Press, Princeton, 2010)

4. E. G. Straus, quoted in: A. Calaprice, ed., The Ultimate Quotable Einstein, p. 344 (Princeton University Press, Princeton, 2010)

5. R. H. Dicke, Dirac's Cosmology and Mach's Principle, Nature 192 (1961) 4801, 440

6. J. D. Barrow and F. Tipler, The Anthropic Cosmological Principle (Oxford University Press, New York 1986). See also: J. D. Barrow, The Constants of Nature (Pantheon Books, New York, 2003)

7. S. Hawking, A Brief History of Time (Bantam Press, London, 1988)

8. P. S. Ehrenfest, In what way does it become manifest in fundamental laws of physics that space has three dimensions?, Proc. Royal Acad. Amsterdam 20, 200 (1917)

9. P. S. Ehrenfest, How do the fundamental laws of physics make manifest that space has three dimensions?, Annalen der Physik Vol. 61 Nr. 5, 440 (1920)

10. F. R. Tangherlini, Schwarzschild field in $n$ dimensions and the dimensionality of space problem, Nuovo Cimento 27, 636 (1963)

11. P.A.R. Ade et al., Planck Collab. 2015 Results XIII, Astronomy and Astrophysics 594, A13 (2016). arXiv:1502.01589

12. S. Weinberg, Anthropic Bound on the Cosmological Constant, Phys. Rev. Lett. Vol. 59 Nr. 22, 2607 (1987)

13. M. Rees, Just Six Numbers. The Deep Forces That Shape The Universe (Basic Books, New York, 2000)

14. S. Hawking, A Brief History of Time (Bantam Press, London, 1988)

15. S. Weinberg, quoted from: G. F. Giudice in: Perspectives on LHC Physics, G. L. Kane, A. Pierce (Eds.), p. 172 (World Scientific, Singapore, 2008) 
16. M. Green, J. Schwarz and E. Witten, Superstring theory, two volumes (Cambridge University Press, 2012).

17. M. Douglas, The statistics of string/M theory vacua, JHEP 0305, 046 (2003). arXiv:hep-th/0303194;

S. Ashok and M. Douglas, JHEP 0401, 060 (2004). arXiv:hep-th/0307049

18. L. Susskind, The anthropic landscape of string theory, arXiv:hep-th/0302219.

19. A. D. Linde, Particle Physics and Inflationary Cosmology (Harwood, Chur, Switzerland, 1990). See also: arXiv:hep-th/0503203

20. For a popular introduction see e.g.: A. Guth, The Inflationary Universe: The Quest for a New Theory of Cosmic Origins (Perseus Basic Books, New York, 1997)

21. S. Weinberg, Living in the Multiverse, in: Universe or Multiverse?, ed. B. Carr (Cambridge University Press 2007). See also: arXiv:hep-th/0511037

22. P. Steinhardt, Big Bang blunder bursts the Multiverse bubble, Nature 510, 9 (June 3, 2014)

23. M. Gleiser, Universe Or universe? It All Depends On The Multiverse. npr commentary, February 20, 2013, www.npr.org/sections/13.7/2013/02/19/172391249/universe-oruniverse-it-all-depends-on-the-multiverse

24. M. Gleiser, The Universe Next Door, npr commentary, January 9, 2013, www.npr.org/blogs/13.7/2013/01/09/168863713/good-news-bad-news-the-universe-nextdoor

25. L. Smolin, The Life of the Cosmos (Oxford University Press, New York, 1997)

26. M. Gell-Mann, quoted by M. Duff in: New Scientist, 30. June 1988, Vol. 118, No. 1619 , p. 70.

27. J. Monod, Chance and Necessity, p. 180 (Alfred Knopf, New York, 1971)

28. G. Ellis, Scientific American, 1 August 2011, 305 38-43 (2011) 